\title{
A FIXED POINT THEOREM FOR MAPPINGS SATISFYING A GENERAL CONTRACTIVE CONDITION OF INTEGRAL TYPE
}

\section{A. BRANCIARI}

Received 30 April 2001

We analyze the existence of fixed points for mappings defined on complete metric spaces $(X, d)$ satisfying a general contractive inequality of integral type. This condition is analogous to Banach-Caccioppoli's one; in short, we study mappings $f: X \rightarrow X$ for which there exists a real number $c \in] 0,1$, such that for each $x, y \in X$ we have $\int_{0}^{d(f x, f y)} \varphi(t) d t \leq$ $c \int_{0}^{d(x, y)} \varphi(t) d t$, where $\varphi:[0,+\infty[\rightarrow[0,+\infty]$ is a Lebesgue-integrable mapping which is summable on each compact subset of $[0,+\infty$ [, nonnegative and such that for each $\varepsilon>0$, $\int_{0}^{\varepsilon} \varphi(t) d t>0$.

2000 Mathematics Subject Classification: 54H25, 47H10.

1. Introduction. The first important result on fixed points for contractive-type mappings was the well-known Banach-Caccioppoli theorem, published for the first time in 1922 in [1] (see also [3]). In the general setting of complete metric spaces this theorem runs as follows (see [9, Theorem 1.2.2]).

THEOREM 1.1. Let $(X, d)$ be a complete metric space, $c \in] 0,1[$, and let $f: X \rightarrow X$ be a mapping such that for each $x, y \in X$,

$$
d(f x, f y) \leq c d(x, y)
$$

then $f$ has a unique fixed point $a \in X$ such that for each $x \in X, \lim _{n \rightarrow+\infty} f^{n} x=a$.

After this classical result Kannan in [4] analyzed a substantially new type of contractive condition. Since then there have been many theorems dealing with mappings satisfying various types of contractive inequalities. Such conditions involve linear and nonlinear expressions (rational, irrational, and of general type). The interested reader who wants to know more about this matter is recommended to go deep into the survey articles by Rhoades [6, 7, 8] and Meszáros [5], and into the references therein.

The aim of this paper is to analyze the existence of fixed points for mappings $f$ defined on a complete metric space $(X, d)$ satisfying a contractive condition of integral type (see (2.1) below).

First we introduce the matter and we present Banach-Caccioppoli fixed point theorem; Section 2 contains the main result. At the end of the paper some remarks and examples concerning this kind of contractions are given.

In the sequel, $\mathbb{N}$ will represent the set of natural numbers (starting from 1 ), $\mathbb{R}$ the set of real numbers, and $\mathbb{R}^{+}$the set of nonnegative real numbers. 
2. Main results. The following theorem is the main result of this paper; the proof, which proceeds by steps, is based on an argument similar to the one used by Boyd and Wong [2, Theorem 1].

THEOREM 2.1. Let $(X, d)$ be a complete metric space, $c \in] 0,1[$, and let $f: X \rightarrow X$ be a mapping such that for each $x, y \in X$,

$$
\int_{0}^{d(f x, f y)} \varphi(t) d t \leq c \int_{0}^{d(x, y)} \varphi(t) d t
$$

where $\varphi:[0,+\infty[\rightarrow[0,+\infty]$ is a Lebesgue-integrable mapping which is summable (i.e., with finite integral) on each compact subset of $[0,+\infty[$, nonnegative, and such that for each $\varepsilon>0, \int_{0}^{\varepsilon} \varphi(t) d t>0$; then $f$ has a unique fixed point $a \in X$ such that for each $x \in X, \lim _{n \rightarrow+\infty} f^{n} x=a$.

\section{ProOF}

STEP 1. We have

$$
\int_{0}^{d\left(f^{n} x, f^{n+1} x\right)} \varphi(t) d t \leq c^{n} \int_{0}^{d(x, f x)} \varphi(t) d t .
$$

This follows immediately by iterating (2.1) $n$ times:

$$
\int_{0}^{d\left(f^{n} x, f^{n+1} x\right)} \varphi(t) d t \leq c \int_{0}^{d\left(f^{n-1} x, f^{n} x\right)} \varphi(t) d t \leq \cdots \leq c^{n} \int_{0}^{d(x, f x)} \varphi(t) d t .
$$

As a consequence, since $c \in] 0,1[$, we further have

$$
\int_{0}^{d\left(f^{n} x, f^{n+1} x\right)} \varphi(t) d t \longrightarrow 0+\text { as } n \longrightarrow+\infty .
$$

STEP 2. We have $d\left(f^{n} x, f^{n+1} x\right) \rightarrow 0$ as $n \rightarrow+\infty$. Suppose that

$$
\lim _{n \rightarrow+\infty} \sup d\left(f^{n} x, f^{n+1} x\right)=\varepsilon>0,
$$

then there exist a $v_{\varepsilon} \in \mathbb{N}$ and a sequence $\left(f^{n_{v}} x\right)_{v \geq v_{\varepsilon}}$ such that $d\left(f^{n_{v}} x, f^{n_{\nu}+1} x\right) \rightarrow \varepsilon>$ 0 as $v \rightarrow+\infty$ and $d\left(f^{n_{v}} x, f^{n_{v}+1} x\right) \geq \varepsilon / 2$ for each $v \geq v_{\varepsilon}$, thus (by Step 1 and the sign of $\varphi$ ) we have the following contradiction:

$$
0=\lim _{v \rightarrow+\infty} \int_{0}^{d\left(f^{n_{\nu}} x, f^{n_{\nu}+1} x\right)} \varphi(t) d t \geq \int_{0}^{\varepsilon / 2} \varphi(t) d t>0 .
$$

STEP 3. For each $x \in X\left(f^{n} x\right)_{n \in \mathbb{N}}$ is a Cauchy sequence, that is

$$
\forall \varepsilon>0 \quad \exists v_{\varepsilon} \in \mathbb{N} \mid \forall m, n \in \mathbb{N}, m>n>v_{\varepsilon} d\left(f^{m} x, f^{n} x\right)<\varepsilon .
$$

Suppose that there exists an $\varepsilon>0$ such that for each $v \in \mathbb{N}$ there are $m_{v}, n_{v} \in \mathbb{N}$, with $m_{v}>n_{v}>v$, such that $d\left(f^{m_{v}} x, f^{n_{v}} x\right) \geq \varepsilon$, then we choose the sequences $\left(m_{v}\right)_{v \in \mathbb{N}}$ and $\left(n_{v}\right)_{v \in \mathbb{N}}$ such that for each $v \in \mathbb{N} m_{v}$ is "minimal" in the sense that $d\left(f^{m_{v}} x, f^{n_{v}} x\right) \geq \varepsilon$ but $d\left(f^{h} x, f^{n_{v}} x\right)<\varepsilon$ for each $h \in\left\{n_{v}+1, \ldots, m_{v}-1\right\}$. 
Now we analyze the properties of $d\left(f^{m_{v}} x, f^{n_{v}} x\right)$ and $d\left(f^{m_{v}+1} x, f^{n_{v}+1} x\right)$. First of all, we have $d\left(f^{m_{v}} x, f^{n_{v}} x\right) \rightarrow \varepsilon+$ as $v \rightarrow+\infty$, in fact by the triangular inequality and Step 2

$$
\begin{aligned}
\varepsilon & \leq d\left(f^{m_{v}} x, f^{n_{v}} x\right) \\
& \leq d\left(f^{m_{v}} x, f^{m_{v}-1} x\right)+d\left(f^{m_{v}-1} x, f^{n_{v}} x\right) \\
& <d\left(f^{m_{v}} x, f^{m_{v}-1} x\right)+\varepsilon \stackrel{v \rightarrow+\infty}{\longrightarrow} \varepsilon+,
\end{aligned}
$$

further there exists $\mu \in \mathbb{N}$ such that for each natural number $v>\mu$, one has $d\left(f^{m_{v}+1} x\right.$, $\left.f^{n_{v}+1} x\right)<\varepsilon$; in fact, if there exists a subsequence $\left(v_{k}\right)_{k \in \mathbb{N}} \subseteq \mathbb{N}$ such that $d\left(f^{m_{v_{k}+1}} x\right.$, $\left.f^{n_{v_{k}}+1} x\right) \geq \varepsilon$, then

$$
\begin{aligned}
\varepsilon \leq & d\left(f^{m_{v_{k}}+1} x, f^{n_{v_{k}}+1} x\right) \leq d\left(f^{m_{v_{k}}+1} x, f^{m_{v_{k}}} x\right) \\
& +d\left(f^{m_{v_{k}}} x, f^{n_{v_{k}}} x\right)+d\left(f^{n_{v_{k}}} x, f^{n_{v_{k}}+1} x\right) \stackrel{k_{k+\infty}}{\longrightarrow} \varepsilon
\end{aligned}
$$

and from (2.1)

$$
\int_{0}^{d\left(f^{m_{v_{k}+1}} x, f^{n_{v_{k}+1}} x\right)} \varphi(t) d t \leq c \int_{0}^{d\left(f^{m_{v_{k}}} x, f^{\left.n_{v_{k}} x\right)}\right.} \varphi(t) d t,
$$

letting now $k \rightarrow+\infty$ in both sides of (2.10), we have $\int_{0}^{\varepsilon} \varphi(t) d t \leq c \int_{0}^{\varepsilon} \varphi(t) d t$ which is a contradiction being $c \in] 0,1$ [ and the integral being positive. Therefore for a certain $\mu \in \mathbb{N}$ one has $d\left(f^{m_{\nu}+1} x, f^{n_{\nu}+1} x\right)<\varepsilon$ for all $\nu>\mu$. Finally, we prove the stronger property that there exist a $\left.\sigma_{\varepsilon} \in\right] 0, \varepsilon\left[\right.$ and a $\nu_{\varepsilon} \in \mathbb{N}$ such that for each $v>\nu_{\varepsilon}(\nu \in \mathbb{N})$ we have $d\left(f^{m_{v}+1} x, f^{n_{v}+1} x\right)<\varepsilon-\sigma_{\varepsilon}$; suppose the existence of a subsequence $\left(v_{k}\right)_{k \in \mathbb{N}} \subseteq \mathbb{N}$ such that $d\left(f^{m_{v_{k}+1}} x, f^{n_{v_{k}}+1} x\right) \rightarrow \varepsilon-$ as $k \rightarrow+\infty$, then from

$$
\int_{0}^{d\left(f^{m_{v_{k}+1}} x, f^{n_{v_{k}+1}} x\right)} \varphi(t) d t \leq c \int_{0}^{d\left(f^{m_{v_{k}}} x, f^{\left.n_{v_{k}} x\right)}\right.} \varphi(t) d t,
$$

letting $k \rightarrow+\infty$, we have again the contradiction that $\int_{0}^{\varepsilon} \varphi(t) d t \leq c \int_{0}^{\varepsilon} \varphi(t) d t$. In conclusion of this step we can prove the Cauchy character of $\left(f^{n} x\right)_{n \in \mathbb{N}}(x \in X)$; in fact for each natural number $v>v_{\varepsilon}\left(v_{\varepsilon}\right.$ as above)

$$
\begin{aligned}
\varepsilon \leq & d\left(f^{m_{v}} x, f^{n_{v}} x\right) \leq d\left(f^{m_{v}} x, f^{m_{v}+1} x\right) \\
& +d\left(f^{m_{v}+1} x, f^{n_{v}+1} x\right)+d\left(f^{n_{v}+1} x, f^{n_{v}} x\right) \\
< & d\left(f^{m_{v}} x, f^{m_{v}+1} x\right)+\left(\varepsilon-\sigma_{\varepsilon}\right)+d\left(f^{n_{v}} x, f^{n_{v}+1} x\right) \stackrel{v \rightarrow+\infty}{\longrightarrow} \varepsilon-\sigma_{\varepsilon},
\end{aligned}
$$

thus $\varepsilon \leq \varepsilon-\sigma_{\varepsilon}$ which is a contradiction. This proves Step 3 .

STEP 4. Existence of a fixed point. Since $(X, d)$ is a complete metric space, there exists a point $a \in X$ such that $a=\lim _{n \rightarrow+\infty} f^{n} x$; further $a$ is a fixed point, in fact suppose that $d(a, f a)>0$, thus

$$
0<d(a, f a) \leq d\left(a, f^{n+1} x\right)+d\left(f^{n+1} x, f a\right) \stackrel{n \rightarrow+\infty}{\longrightarrow} 0
$$


in fact both $d\left(a, f^{n+1} x\right)$ and $d\left(f^{n+1} x, f a\right)$ converge to 0 as $n \rightarrow+\infty$ : for the first one it is obvious, while for the second one we have

$$
\int_{0}^{d\left(f^{n+1} x, f a\right)} \varphi(t) d t \leq c \int_{0}^{d\left(f^{n} x, a\right)} \varphi(t) d t \stackrel{n \rightarrow+\infty}{\longrightarrow} 0 .
$$

Now if $d\left(f^{n+1} x, f a\right)$ does not converge to 0 as $n \rightarrow+\infty$, then there exists a subsequence $\left(f^{n_{v}+1} x\right)_{v \in \mathbb{N}} \subseteq\left(f^{n+1} x\right)_{n \in \mathbb{N}}$ such that $d\left(f^{n_{v}+1} x, f a\right) \geq \varepsilon$ for a certain $\varepsilon>0$; thus we have the following contradiction:

$$
0<\int_{0}^{\varepsilon} \varphi(t) d t \leq \int_{0}^{d\left(f^{n_{\nu}+1} x, f a\right)} \varphi(t) d t \stackrel{v \rightarrow+\infty}{\longrightarrow} 0 .
$$

STEP 5. Uniqueness of the fixed point. Suppose that there are two distinct points $a, b \in X$ such that $f a=a$ and $f b=b$, then by (2.1) we have the contradiction

$$
0<\int_{0}^{d(a, b)} \varphi(t) d t=\int_{0}^{d(f a, f b)} \varphi(t) d t \leq c \int_{0}^{d(a, b)} \varphi(t) d t<\int_{0}^{d(a, b)} \varphi(t) d t .
$$

This final step also proves that for each $x \in X, \lim _{n \rightarrow+\infty} f^{n} x=a=f a$. The proof is thus completed.

3. Final remarks and examples. In this section, we give some remarks and examples concerning these contractive mappings of integral type, which clarify the connection between our result and the classical ones.

REMARK 3.1. Theorem 2.1 is a generalization of the Banach-Caccioppoli principle, letting $\varphi(t)=1$ for each $t \geq 0$ in (2.1), we have

$$
\int_{0}^{d(f x, f y)} \varphi(t) d t=d(f x, f y) \leq c d(x, y)=c \int_{0}^{d(x, y)} \varphi(t) d t
$$

thus a Banach-Caccioppoli contraction also satisfies (2.1). The converse is not true as we will see in Example 3.6.

REMARK 3.2. We have used the idea of contractive mappings of integral type to generalize Banach-Caccioppoli's theorem, but in a similar way we can generalize other results also related to contractive conditions of some kind, such as the ones contained in $[5,6,7,8]$.

REMARK 3.3. Theorem 2.1 is no more true if we admit zero value almost everywhere near zero for the mapping $\varphi$; we show it with the following example. In a similar way, we cannot admit negative value for $\varphi$, as in Example 3.5.

EXAMPLE 3.4. Let $f: \mathbb{N} \rightarrow \mathbb{N}$ and $\varphi: \mathbb{R}^{+} \rightarrow \mathbb{R}^{+}$be defined by

$$
f x \stackrel{\text { def }}{=}\left\{\begin{array} { l l } 
{ 1 } & { \text { if } x \neq 1 , } \\
{ 2 } & { \text { if } x = 1 ; }
\end{array} \quad \varphi ( t ) \stackrel { \text { def } } { = } \left\{\begin{array}{ll}
e^{1 /(1-t)} & \text { if } t>1, \\
0 & \text { if } t \in[0,1],
\end{array}\right.\right.
$$

and let $d: \mathbb{N}^{2} \rightarrow \mathbb{R}^{+}$be the Euclidean metric restricted to $\mathbb{N}$ (so that $(\mathbb{N}, d$ ) becomes a complete metric space). Now, since for each $x, y \in \mathbb{N}, d(f x, f y) \leq 1$, we have for an 
arbitrary $c \in] 0,1[$

$$
\int_{0}^{d(f x, f y)} \varphi(t) d t \leq \int_{0}^{1} \varphi(t) d t=0 \leq c \int_{0}^{d(x, y)} \varphi(t) d t
$$

thus (2.1) is satisfied for all $c \in] 0,1$, but $f$ has no fixed points.

EXAMPLE 3.5. Let $f: \mathbb{R}^{+} \rightarrow \mathbb{R}^{+}$be defined by $f x:=x+1$ and let $\varphi \equiv-1$, then for an arbitrary $c \in$ ]0,1 [ we have ( $d$ is the Euclidean distance function)

$$
\int_{0}^{d(f x, f y)} \varphi(t) d t=-d(f x, f y)=-d(x, y) \leq-c d(x, y)=c \int_{0}^{d(x, y)} \varphi(t) d t
$$

thus (2.1) is satisfied with $\varphi \equiv-1$ and for all $c \in] 0,1$, but $f$, being a translation on $\mathbb{R}^{+}$, has no fixed points.

EXAMPLE 3.6. Let $X:=\{1 / n \mid n \in \mathbb{N}\} \cup\{0\}$ with metric induced by $\mathbb{R}: d(x, y):=$ $|x-y|$, thus, since $X$ is a closed subset of $\mathbb{R}$, it is a complete metric space. We consider now a mapping $f: X \rightarrow X$ defined by

$$
f x \stackrel{\text { def }}{=} \begin{cases}\frac{1}{n+1} & \text { if } x=\frac{1}{n} n \in \mathbb{N} \\ 0 & \text { if } x=0\end{cases}
$$

then it satisfies (2.1) with $\varphi(t)=t^{1 / t-2}[1-\log t]$ for $t>0, \varphi(0)=0$, and $c=1 / 2$. In this context one has $\int_{0}^{T} \varphi(t) d t=\tau^{1 / r}$, so that (2.1), for $x \neq y$, is equivalent to

$$
d(f x, f y)^{1 / d(f x, f y)} \leq c d(x, y)^{1 / d(x, y)} .
$$

The next step is thus the proof of the validity of (3.6): let $m, n \in \mathbb{N}$ with $m>n$ and let $x=1 / n, y=1 / m$, then we have

$$
\begin{aligned}
d(f x, f y)^{1 / d(f x, f y)} & =\left|\frac{1}{n+1}-\frac{1}{m+1}\right|^{1 /|1 /(n+1)-1 /(m+1)|} \\
& =\left[\frac{m-n}{(n+1)(m+1)}\right]^{(n+1)(m+1) /(m-n)},
\end{aligned}
$$

while on the other hand,

$$
d(x, y)^{1 / d(x, y)}=\left|\frac{1}{n}-\frac{1}{m}\right|^{1 /|1 / n-1 / m|}=\left[\frac{m-n}{n m}\right]^{n m /(m-n)} .
$$

We now show that

$$
\left[\frac{m-n}{(n+1)(m+1)}\right]^{(n+1)(m+1) /(m-n)} \leq \frac{1}{2}\left[\frac{m-n}{n m}\right]^{n m /(m-n)},
$$

or equivalently

$$
\left[\frac{m-n}{(n+1)(m+1)}\right]^{(n+m+1) /(m-n)} \cdot\left[\frac{n m}{(n+1)(m+1)}\right]^{n m /(m-n)} \leq \frac{1}{2} .
$$


This last inequality is indeed true; analyzing the first member, we have

$$
\left[\frac{n m}{(n+1)(m+1)}\right]^{n m /(m-n)} \leq 1,
$$

since $n m<(n+1)(m+1)$ and $n m /(m-n)>0$, and also

$$
\left[\frac{m-n}{(n+1)(m+1)}\right]^{(n+m+1) /(m-n)} \leq \frac{1}{2}
$$

the base at the first member of (3.12) is lesser than $1 / 2$ (since for all $m, n \in \mathbb{N}$ we have $m \leq 3 n+n m+1$, and thus $2(m-n) \leq(n+1)(m+1))$, while the exponent is greater than 1 (since for all $m, n \in \mathbb{N}, n+m+1>m-n$ is trivially satisfied). On the other hand, taking $x=1 / n(n \in \mathbb{N})$ and $y=0$ we have

$$
d(f x, f y)^{1 / d(f x, f y)}=\left[\frac{1}{n+1}\right]^{n+1} \leq \frac{1}{2}\left[\frac{1}{n}\right]^{n}=\frac{1}{2} d(x, y)^{1 / d(x, y)}
$$

because for each $n \in \mathbb{N}$ we have

$$
\left[\frac{n}{n+1}\right]^{n} \cdot \frac{1}{n+1} \leq \frac{1}{2}
$$

since $n /(n+1)<1$ and $1 /(n+1) \leq 1 / 2$.

Therefore such mapping $f$ satisfies condition (3.6) with $c=1 / 2$ and therefore (2.1) with the same $c$ and for $\varphi$ defined by $\varphi(t)=t^{1 / t-2}[1-\log t]$ for $t>0$ and $\varphi(0)=0$, but

$$
\sup _{\{x, y \in X \mid x \neq y\}} \frac{d(f x, f y)}{d(x, y)}=1,
$$

thus it is not a Banach-Caccioppoli contraction.

\section{REFERENCES}

[1] S. Banach, Sur les opérations dans les ensembles abstraits et leur application aux équations intégrales, Fund. Math. 3 (1922), 133-181 (French).

[2] D. W. Boyd and J. S. W. Wong, On nonlinear contractions, Proc. Amer. Math. Soc. 20 (1969), 458-464.

[3] R. Caccioppoli, Un teorema generale sull' esistenza di elementi uniti in una trasformazione funzionale, Rend. Accad. dei Lincei 11 (1930), 794-799 (Italian).

[4] R. Kannan, Some results on fixed points, Bull. Calcutta Math. Soc. 60 (1968), 71-76.

[5] J. Meszáros, A comparison of various definitions of contractive type mappings, Bull. Calcutta Math. Soc. 84 (1992), no. 2, 167-194.

[6] B. E. Rhoades, A comparison of various definitions of contractive mappings, Trans. Amer. Math. Soc. 226 (1977), 257-290.

[7] Contractive definitions revisited, Topological Methods in Nonlinear Functional Analysis (Toronto, Ont., 1982), Contemp. Math., vol. 21, American Mathematical Society, Rhode Island, 1983, pp. 189-205.

[8] _ Contractive definitions, Nonlinear Analysis, World Science Publishing, Singapore, 1987, pp. 513-526.

[9] D. R. Smart, Fixed Point Theorems, Cambridge University Press, London, 1974.

A. BRANCIARI: VIALE MARTIRI DELLA LibERTÀ 20, 62100 MACERATA, ITALY

E-mail address: abranci@i ibero . it 


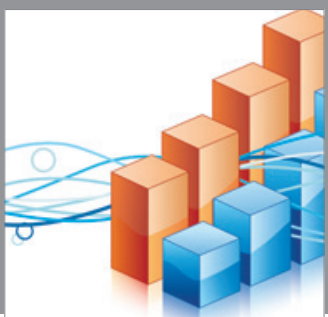

Advances in

Operations Research

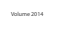

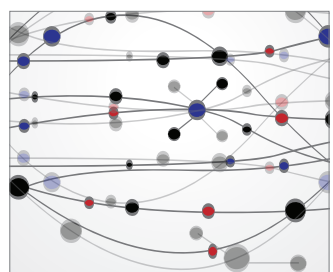

\section{The Scientific} World Journal
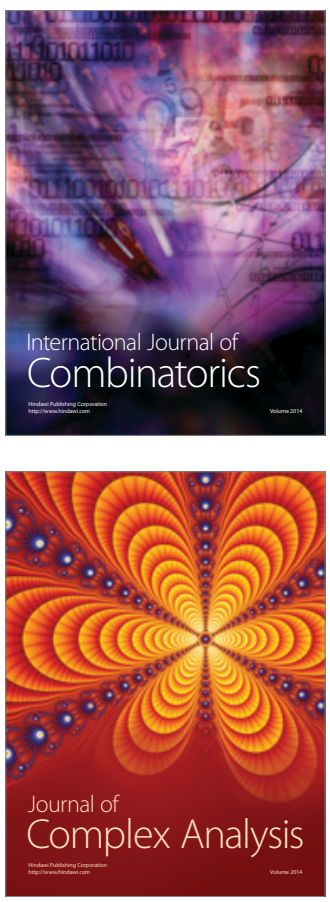

International Journal of

Mathematics and

Mathematical

Sciences
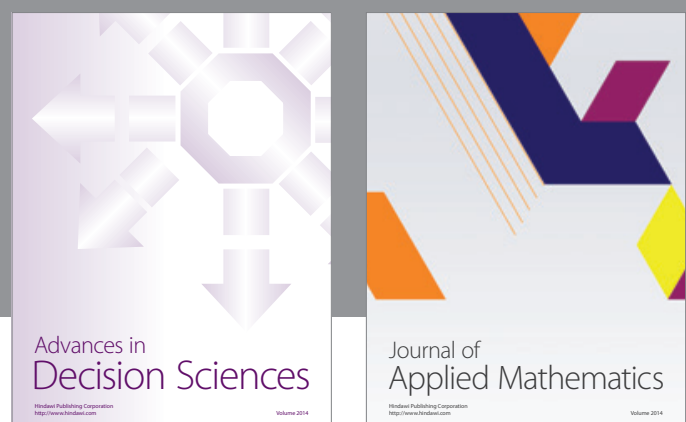

Journal of

Applied Mathematics
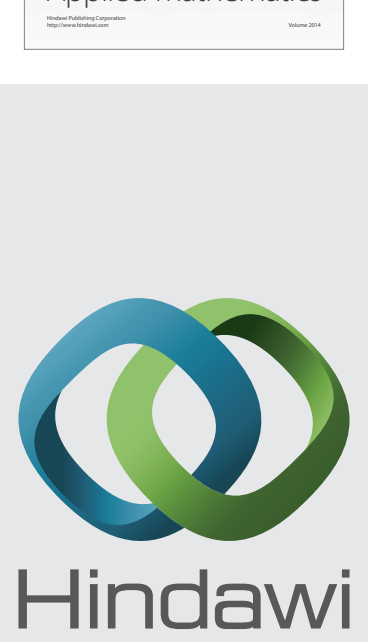

Submit your manuscripts at http://www.hindawi.com
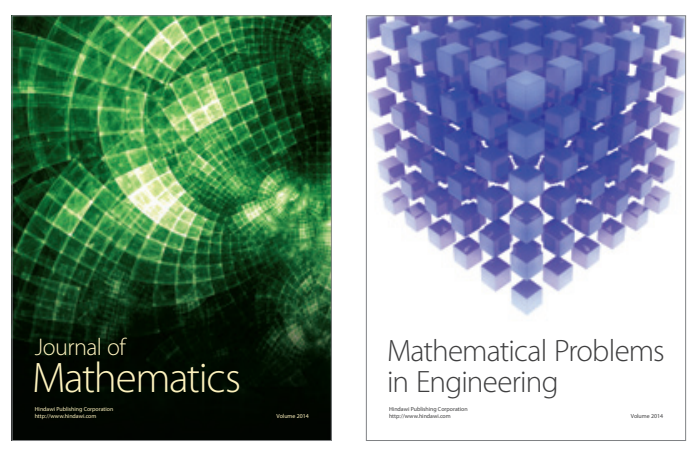

Mathematical Problems in Engineering
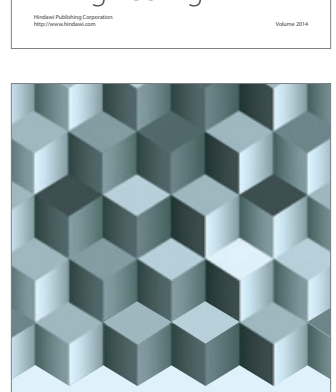

Journal of

Function Spaces
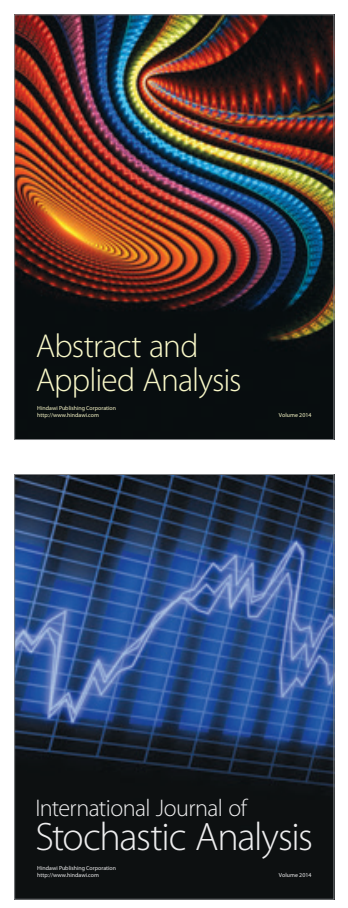

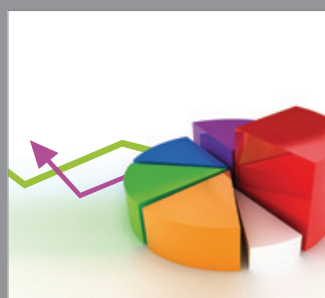

ournal of

Probability and Statistics

Promensencen
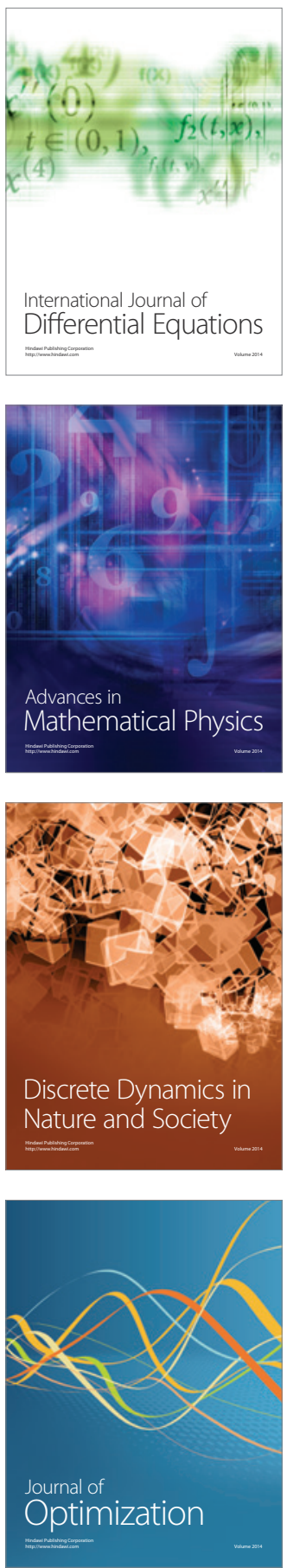